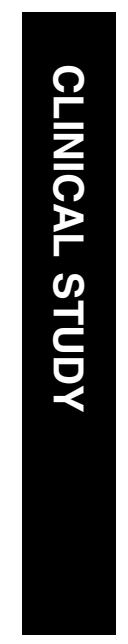

Glaucoma Service, Moorfield's Eye Hospital, London, UK

Correspondence:

PA Andreou, Broomfield Hospital, Chelmsford, Essex CM1 7HJ, UK

Received: 23 May 2005 Accepted in revised form: 29 January 2006 Published online: 12 May 2006

Presented at the American Academy of Ophthalmology Meeting October 2002, $\mathrm{FL}$, USA

\title{
A comparison of HRT II and GDx imaging for glaucoma detection in a primary care eye clinic setting
}

\begin{abstract}
Purpose To evaluate the performance of the HRT II (Heidelberg retinal tomograph) and GDx (glaucoma detection) retinal nerve fibre analyzer in GDx when used in the primary care eye clinic setting for glaucoma screening. Patients and Methods The study was prospective, cross-sectional, and hospitalbased. One-hundred and twelve patients, 59 women and 53 men with a mean age of 57.8 years (range 18-85 years), had consecutive HRT II disc imaging and GDx retinal nerve fiber layer analysis. The Moorfield's regression classification and the 'GDx number' were used to predict the likelihood of glaucoma. A separate clinician, masked to the instrument results determined a definitive diagnosis, based on clinical examination. The extent of agreement between instrument prediction and the clinician diagnosis of glaucoma was examined by generating sensitivity and specificity tables.

Results The HRT II had a sensitivity of 0.79 (95\% CI: $0.60-0.92$ ) and a specificity of 0.70 (95\% CI: 0.60-0.78). The positive predictive value of the HRT II was 0.43 (95\% CI: 0.29-0.57). Using a GDx number of 50 as 'cutoff' for glaucoma detection, the GDx had a sensitivity of 0.80 (95\% CI: $0.59-0.93)$ and a specificity of 0.72 (95\% CI: $0.61-0.80)$, with a positive predictive value of 0.43 (95\% CI: $0.28-0.59$ ). Conclusions For glaucoma detection, neither the HRT II nor the GDx are effective as stand-alone screening devices in the primary care setting.

Eye (2007) 21, 1050-1055; doi:10.1038/sj.eye.6702394; published online 12 May 2006
\end{abstract}

Keywords: screening; nerve fibre layer; optic disc
PA Andreou, SS Wickremasinghe, RH Asaria, E Tay and WA Franks

\section{Introduction}

Glaucoma is a disease characterized by the progressive loss of retinal ganglion cells, corresponding histologically to a decrease in thickness of the nerve fibre layer. Functional loss in glaucoma is manifested by visual field loss when sufficient nerve fibres have been lost either focally or diffusely and show corresponding changes in optic disc topography. An estimated $40-50 \%$ of nerve fibres may be lost before functional visual loss occurs. Consequently, conventional perimetric testing may not be sensitive enough to detect early disease or subtle progression and lead to delays in starting or changing treatments.

Both retinal nerve fibre layer (RNFL) thickness disturbances and optic disc damage have been shown to precede visual loss ${ }^{1-4}$ so that objective assessment of these parameters may help physicians detect and monitor glaucoma earlier.

Over the last few years, a number of new methods have been developed to assess the RNFL and the optic disc, by using different properties of light. Previous studies have shown that, despite considerable overlap in the optic disc and RNFL measurements, statistically significant differences exist between normal and glaucomatous eyes. ${ }^{5-8}$ There are, however, conflicting reports regarding the ability of these methods to discriminate between normal and glaucomatous eyes.

In the primary care setting, the differentiation of patients with definite glaucoma and those who are normal is usually straightforward. It is the ability to detect those with glaucoma from patients with slightly suspicious disc features or mildly elevated intraocular pressures (IOP) that often prove more difficult. Hence, there may be a tendency for over referral or more alarmingly 
under referral. Methods that would help in this differentiation would be of great benefit. Hence, this study was designed to compare the ability of a scanning laser ophthalmoscope, Heidelberg retinal tomograph II (HRT II) with a scanning laser polarimeter, GDx nerve fibre layer analyzer (GDx) in detecting those with early glaucoma from a group of suspected glaucoma patients.

\section{Methods}

\section{Subjects}

All new patient referrals to the primary eye care clinic at Mile End Community Hospital between the dates of April and June 2001 with a possible diagnosis of glaucoma were enrolled in to the study. All the patients were referred by local optometrists after an examination including, IOP measurement by either contact or noncontact tonometry and/or perimetry and clinical optic disc assessment. Referral was made on the basis of optic discs cupping suspicious of glaucoma, raised IOPs, or visual field defects.

Informed consent was obtained from all patients and Ethics committee (Moorfield's Eye Hospital) approval was attained.

Following recruitment, both eyes of each subject were imaged using both the HRT II and GDx nerve fibre layer analyzer. Imaging was carried out by experienced technicians, who obtained three satisfactory scans from each eye. The order of measurement was randomly determined by the availability of the instrument.

A glaucoma consultant specialist blinded to both the HRT and GDx measurements, assessed all the patients and assigned a clinical diagnosis based on an ophthalmic examination, including slit-lamp biomicroscopy, Goldmann applanation tonometry, gonioscopy, dilated fundus examination, and a reliable 24-2 SITA standard Humphrey field analysis. (Reliability of the field was assessed using the following criteria, less than $20 \%$ fixation losses, and up to a maximum of $30 \%$ falsepositive and false-negative errors).

Using the clinical and perimetric information, the glaucoma specialist categorized each patient into one of the following groups, 'definite glaucoma', 'glaucoma suspect' (GS), 'ocular hypertension' (OHT), 'nonglaucomatous optic neuropathy', or 'normal'. The diagnosis of glaucoma was made on the basis of optic disc cupping, greater than 0.4 or asymmetry of vertical cup-disc ratio by more than 0.2 with a corresponding visual field defect. A glaucomatous visual field defect was defined if $4 / 6$ of the following criteria were met on 24-2 SITA, (i) a pattern standard deviation (PSD) worse than the normal 1\% level, (ii) a glaucoma hemifield test (GHT) 'outside normal limits' (ONL), (iii) one hemifield cluster worse than the normal $1 \%$ level, (iv) two hemifield clusters worse than the $5 \%$ level, (v) four abnormal $(P<0.05)$ locations, (vi) five abnormal locations $(P<0.05)$ on the pattern deviation probability plot. OHT was defined as an IOP of greater than $21 \mathrm{mmHg}$, in the absence of glaucomatous optic disc or perimetric changes. GS were patients with some, but not all of the criteria needed for a diagnosis of definite glaucoma. Healthy eyes were defined as having IOPs $21 \mathrm{mmHg}$ or less, with no history of elevated IOP. These eyes had intact rims, no evidence of haemorrhage, notching, excavation, or RNFL defect and symmetrical optic discs based on clinical examination. Nonglaucomatous optic neuropathy was diagnosed in patients regardless of IOP. These subjects had optic nerve head changes inconsistent with the characteristic cupping or visual field defects associated with glaucomatous optic neuropathy.

A separate ophthalmologist, masked to the patients' clinical examination analysed the data from the HRT II and GDx devices for possible prediction of glaucoma.

HRT II disc imaging was graded as 'normal', 'borderline', and 'ONL' based on the Moorfield's regression coefficient. For GDx neural peripapillary imaging, 'normal' and 'abnormal' results were defined in terms of a GDx number of 50 (If the subject fell above this value, they were considered to have an abnormal result and visa versa.)

\section{Instrumentation}

\section{Confocal scanning laser ophthalmoscope}

The HRT II (Heidelberg Engineering, Heidelberg, Germany) utilizes diode technology to produce topographical measurements of the optic disc. The image is derived from 32 optical sections at consecutive focal depth planes. Three $15^{\circ}$ field-of-view scans, judged to be of acceptable quality, were obtained for each eye. A mean topographic image based on these scans was created using HRT software version 1.11. A technician outlined the optic disc margin on the mean topographic image while observing the stereoscopic photographs of the optic disc. The Moorfield's regression classification ${ }^{9}$ (obtained using the $99 \%$ prediction interval from the linear regression between the optic disc area and the log of the neuroretinal rim area) was used to give a categorical analysis of the disc. Classification of the disc was into three categories, ONL, within normal limits (WNL), and borderline (B). We used ONL as a diagnosis of glaucoma.

\section{Scanning laser polarimetry}

The GDx nerve fibre layer analyzer (Laser Diagnostic Technologies, San Diego, CA, USA) uses scanning laser technology coupled with an integrated polarization 
modulator to provide a retardation map of the peripapillary retina, based on the birefringent properties of the RNFL. Measurement of the retardation of light that has double passed the birefringent fibres of the RNFL is used to construct an image, each pixel corresponding to the retardation value at its location. The GDx softwareprovided parameter utilized in this study was the GDx number $^{10,11}$ (a neural network assessment of glaucoma likelihood). The number generated is based on the analysis of the image pixels. The values vary from 0 to 100, with higher values more likely to represent glaucoma. (The GDx manual suggests that scores below 30 represent a normal nerve fibre layer, scores between 30 to 80 suggest a suspicion of glaucoma, whereas those greater than 80 being highly suggestive of glaucoma).

Three scans of acceptable quality were obtained for each eye, and a mean retardation map comprising each of the scans was created using the GDx software version 2.0.09. The optic disc margin was outlined based on the mean retardation image by a technician. A corneal compensator was not utilized, as this software was not available at the time the study was performed.

\section{Statistical analysis}

In order to determine sensitivity and specificity, normal and abnormal were defined such that, only those who were diagnosed with definite glaucoma were considered as glaucomatous, all other categories including, GS and OHT, were considered as nonglaucomatous.

In terms of instrumentation, ONL was considered as abnormal (B and WNL were analysed as being normal) with the HRT II.

The optimal GDx number for the 'cutoff' between 'normal' and 'abnormal' was determined by the use of receiver operating characteristic (ROC) curves. Table 1 gives the sensitivity, specificity, and area under the ROC curve values at different GDx scores in terms of agreement with the clinician's diagnosis. Fifty was

Table 1 Sensitivity, specificity, and area under the ROC curve at different values of GDx number for agreement with the clinician's diagnosis

\begin{tabular}{lccc}
\hline $\begin{array}{l}\text { Cutoff } \\
\text { value }\end{array}$ & $\begin{array}{c}\text { Sensitivity } \\
(95 \% \text { CI })\end{array}$ & $\begin{array}{c}\text { Specificity } \\
(95 \% \text { CI })\end{array}$ & $\begin{array}{c}\text { Area under } \\
\text { ROC curve }\end{array}$ \\
\hline 30 & $0.96(0.80-1.00)$ & $0.50(0.39-0.60)$ & 0.73 \\
35 & $0.92(0.74-0.99)$ & $0.58(0.47-0.68)$ & 0.75 \\
40 & $0.80(0.59-0.72)$ & $0.62(0.52-0.72)$ & 0.71 \\
45 & $0.80(0.59-0.93)$ & $0.66(0.56-0.76)$ & 0.73 \\
50 & $0.80(0.59-0.93)$ & $0.72(0.61-0.80)$ & 0.76 \\
55 & $0.68(0.46-0.85)$ & $0.78(0.68-0.86)$ & 0.73 \\
60 & $0.56(0.35-0.76)$ & $0.82(0.73-0.89)$ & 0.69 \\
\hline
\end{tabular}

$\mathrm{CI}$, confidence interval; ROC, receiver operating characteristic. determined to be the best 'cutoff' as it gave the greatest area under the ROC curve.

Having determined normal and abnormal results for each test, sensitivity and specificity tables were generated comparing the result from each imaging device to the clinician's diagnosis (gold standard).

Paired $t$-tests were used to compare the GDx number obtained in glaucoma patients as compared to the other categories, whereas $\chi^{2}$ tests were used to evaluate the categorical data from the HRT, comparing glaucoma patients as diagnosed clinically to the other categories. Linear regression analysis was used to explore whether age was a determinant of the GDx number.

The kappa statistics was utilized to ascertain agreement between the devices and the clinician's diagnosis.

Statistical analysis was carried out using SPSS Version 10 (SPSS Inc., Chicago, IL, USA).

\section{Results}

Over the 3-month study period, 112 patients were recruited into the study. There were 59 women and 53 men with a mean age of 57.8 years (range 18-85 years).

Satisfactory disc images were obtained in 106 patients with HRT II and 99 patients with the GDx (Failure to obtain adequate images with both instruments was due to excessive head movement during image acquisition). A clinical diagnosis of glaucoma was made in 15 left and 14 right eyes of 23 patients. The patient demographics are shown in Table 2 . The only significant difference in mean age between the races was between Caucasians and Asians with Caucasians having a mean age of $60.7 \pm 12.8$ years as compared to $43.3 \pm 13.2$ years in Asians, $P<0.001$.

\section{Clinician analysis}

Table 3 demonstrates the diagnosis given by glaucoma specialist after clinical examination and perimetry. The only significant difference in mean age between the different diagnoses, was that those with glaucoma were older than those without, $P=0.003$.

\section{Heidelberg retinal tomograph II}

Moorfield's regression classification was used to determine whether the optic nerve head was likely to be glaucomatous. Table 4 illustrates the data from the study. A $\chi^{2}$ analysis reveals that there was a significant relationship between the diagnosis of glaucoma with the classification of ONL and also a significant relationship between the WNL and the exclusion of glaucoma, $P<0.001$. There was no significant change in the 
Table 2 Demographics of study population

\begin{tabular}{lccccc}
\hline Race & Mean age $\pm S D$ (years) & Range (years) & Male female ratio & Number/ (\%) & Significance (P) \\
\hline Caucasian & $60.7 \pm 12.8$ & $26-85$ & $1.0: 1.7$ & $67(59.8)$ & - \\
Asian & $43.3 \pm 13.2$ & $18-68$ & $1.0: 1.5$ & $13(11.6)$ & $<0.001$ \\
Black & $57.0 \pm 11.9$ & $35-81$ & $1.0: 1.5$ & $26(23.2)$ & 0.08 \\
Others & $60.0 \pm 12.5$ & $40-70$ & $1.0: 1.0$ & $6(5.4)$ & 0.82 \\
\hline
\end{tabular}

Definitions: Caucasian (Northern Europeans), Asian (Indian subcontinent extraction), black (African or Afro-Caribbean origin), others (Chinese, Mediterranean).

$P$-values indicate difference in mean age between Caucasians and other races.

Table 3 Numbers of eyes assigned to a specific diagnosis by glaucoma specialist

\begin{tabular}{|c|c|c|c|c|}
\hline Diagnosis & Right eyes/ (\%) & Left eyes/ (\%) & Mean $( \pm S D)$ age (years) & Significance $(\mathrm{P})$ \\
\hline Normal & $27(24.1 \%)$ & $27(24.1 \%)$ & $56.2 \pm 10.6$ & - \\
\hline Ocular hypertension & $23(20.5)$ & $23(20.5)$ & $59.6 \pm 13.6$ & 0.17 \\
\hline Nonglaucomatous ON & $7(6.3 \%)$ & $9(8.0 \%)$ & $54.8 \pm 12.6$ & 0.71 \\
\hline Glaucoma suspect & $34(30.3 \%)$ & $35(31.3 \%)$ & $54.0 \pm 15.3$ & 0.37 \\
\hline Definite glaucoma & $14(12.5 \%)$ & $15(13.4 \%)$ & $63.6 \pm 13.0$ & 0.003 \\
\hline No data & $7(6.3 \%)$ & $3(2.7 \%)$ & & \\
\hline Total & 112 & 112 & & \\
\hline
\end{tabular}

$P$-values indicate difference in mean age between Caucasians and other races.

Table 4 Distribution of patients using GDx and HRT II, by eye

\begin{tabular}{|c|c|c|c|c|c|c|c|}
\hline \multirow[t]{2}{*}{ Test } & \multirow[t]{2}{*}{ Laterality } & \multirow[t]{2}{*}{ Test result } & \multicolumn{5}{|c|}{ Clinician's diagnosis } \\
\hline & & & Glaucoma & Glaucoma suspect & OHT & Normal & NGON \\
\hline \multirow[t]{4}{*}{ GDx } & \multirow[t]{2}{*}{ Right } & $<50$ & 2 & 9 & 7 & 16 & 3 \\
\hline & & $>50$ & 11 & 5 & 3 & 4 & 1 \\
\hline & \multirow[t]{2}{*}{ Left } & $<50$ & 5 & 10 & 6 & 15 & 2 \\
\hline & & $>50$ & 9 & 6 & 3 & 3 & 2 \\
\hline \multirow[t]{6}{*}{ HRT II } & \multirow[t]{3}{*}{ Right } & WNL & 1 & 5 & 6 & 15 & 2 \\
\hline & & Borderline & 2 & 4 & 1 & 4 & 0 \\
\hline & & ONL & 12 & 7 & 1 & 4 & 2 \\
\hline & \multirow[t]{3}{*}{ Left } & WNL & 1 & 3 & 6 & 14 & 1 \\
\hline & & Borderline & 3 & 7 & 0 & 2 & 1 \\
\hline & & ONL & 12 & 9 & 3 & 3 & 2 \\
\hline
\end{tabular}

WNL, within normal limits; ONL, outside normal limits; OHT, ocular hypertension; NGON, nonglaucomatous optic neuropathy.

Categorization based on the GDx number and the HRT regression coefficient.

proportions of HRT classification with age, $P=0.32$ or ethnicity, $P=0.19$.

The sensivities, specificities, and positive predictive values for the HRT II are summarized in Table 5.

\section{GDx nerve fibre layer analyzer}

There was a significant difference in GDx number between those individuals diagnosed by the glaucoma specialist as being normal $(26.8 \pm 19.4)$ and those with definite glaucoma $(54.0 \pm 25.2)$, GS $(39.6 \pm 22.2)$, and those with OHT $(36.2 \pm 22.4)$. The $P$-values were $P<0.001$,
0.002 , and 0.04 , respectively. There was no difference between the GDx number in nonglaucomatous optic neuropathy $(33.8 \pm 21.3)$ as compared to normals, $P=0.10$. The differences in the demographics of our patients did not significantly affect the GDx number. Linear regression analysis revealed no change in GDx number with increasing age, $r=0.09, P=0.20$. There was no difference in GDx number between the genders $(P=0.40)$ or between Caucasians and other races (Asians, $P=0.11$, Black patients, $P=0.20$, and others, $P=0.66$ ).

The proportions of patients with normal and elevated GDx scores are presented in Table 4. At this level, the 
Table 5 Predictive values for GDx and HRT II as screening tools in the detection of glaucoma, by eye

\begin{tabular}{llcrr}
\hline Test & Laterality & Sensitivity $(95 \% \mathrm{CI})$ & Specificity $(95 \% \mathrm{CI})$ & $P P V(95 \%$ CI $)$ \\
\hline GDx & Right & $0.92(0.61-1.00)$ & $0.73(0.58-0.85)$ & $0.46(0.26-0.67)$ \\
& Left & $0.69(0.39-0.91)$ & $0.70(0.55-0.83)$ & $0.39(0.20-0.61)$ \\
& All & $0.80(0.59-0.93)$ & $0.72(0.61-0.80)$ & $0.43(0.28-0.59)$ \\
HRT II & Right & & & \\
& Left & $0.79(0.49-0.95)$ & $0.73(0.58-0.84)$ & $0.44(0.24-0.65)$ \\
& All & $0.80(0.52-0.96)$ & $0.67(0.52-0.79)$ & $0.41(0.24-0.61)$ \\
& $0.79(0.60-0.92)$ & $0.70(0.60-0.78)$ & $0.43(0.29-0.57)$ \\
\hline
\end{tabular}

PPV, positive predictive value.

sensitivities, specificities, and positive predictive values are summarized in Table 5.

\section{Agreement}

The kappa test ratio indicated that the agreement between GDx and HRT was observed in 59\% of patients. There was agreement between the clinician's diagnosis and the GDx in $69 \%$ and HRT in $67 \%$ of cases.

\section{Discussion}

In this study, we used the neural network output of the GDx, the GDx number, and the Moorfield's regression coefficient of the HRT II to determine the effectiveness of these instruments to screen for glaucoma in a primary care clinic. The results of each instrument were compared to a clinician's diagnosis, which was used as the 'gold standard'.

Our data were consistent with other studies in that, the GDx number correlated well with the diagnosis of the patient. ${ }^{10,12}$ In patients with definite glaucoma, the GDx number was significantly higher than in those who were diagnosed as normal. Those patients with a diagnosis of GS or OHT had intermediate GDx numbers. Similarly the HRT II classification correlated well with the diagnosis and exclusion of glaucoma.

Both the GDx and HRT II were able to detect patients with glaucoma. The sensitivities were 0.80 and 0.79 , respectively. The ability to accurately exclude those without glaucoma (specificity) was not as good in either instrument, although the HRT II was better than the GDx, 0.70 as compared to 0.72 . The positive predictive value (PPV) for each test was very low, with only $43 \%$ of eyes that were classified as abnormal with each test, being clinically determined to be glaucoma.

Our results are broadly similar to previous studies assessing the GDx number in predicting glaucoma. Widely varying sensitivities (0.64-0.96) and specificities $(0.74-0.96)^{10,12-16}$ have been reported.

Similarly, the HRT has quoted sensitivities of $0.42-0.88$, and specificities of $0.84-0.90 .6,16$
The differences in values between studies are probably a reflection of different methodologies, especially the recruitment of subjects. In our study, patients were recruited from those where there was only a suspicion of glaucoma, without a confirmed diagnosis, whereas most other studies used preselected patients with a definite diagnosis.

This led to the GS or OHT categories, where although fitting certain criteria for glaucoma, clinically, the diagnosis remained unconfirmed at the time of the study as the glaucoma diagnosis required a corresponding visual field defect.

As a result, the sensitivity, specificity, and predictive calculations may have been affected. This is because some of these patients may have gone on to develop glaucoma in the future, but in our analysis, they were assumed not to have glaucoma. It is interesting to note that the mean GDx number was significantly higher in GS and OHT patients as compared to nonglaucoma patients, a finding that has been reported previously. ${ }^{10,12}$ It may be that a sizeable proportion of these patients have early glaucoma (not detectable perimetrically), which is consistent with the theory that structural changes precede the earliest detectable visual field defects in glaucoma. ${ }^{1-4}$ Another reason for differences in sensitivity and specificity values are the criteria used for determining a positive or negative result. We used the GDx number and HRT regression classification because as a screening procedure the criteria should be as simple as possible to attain but yet give good reliability, other groups have used specific parameters of the optic disc topography or different 'cutoffs' in GDx number between normal and abnormal.

Limitations of our study include its relatively small sample size leading to only 29 eyes with definite glaucoma. Although there were differences in the mean age of glaucoma vs nonglaucoma patients, we feel that this difference was not a major factor in determining the result obtained by the GDx and HRT II, as GDx number and proportions of HRT regression classification were shown not to change with age. Several reports have described inaccuracy within GDx measurements as a 
result of an inadequate corneal compensation procedure. ${ }^{17,18}$ It has been suggested that this effect may be compensated for by post hoc methods. ${ }^{19,20}$ As with many other studies, we did not allow for this correction, which have also influenced our overall results, although comparisons between groups should not have been greatly impaired. Selection bias introduced, owing to the recruitment of patients being referred as suspects for glaucoma as opposed to a heterogeneous population normally seen in the community. Consequently, the number of normal subjects is likely to be lower than that seen in the normal primary care setting.

In conclusion, although there was good agreement between both imaging devices and the glaucoma specialist, however, neither instrument performs well at their current level of development as a screening tool for glaucoma in a primary care setting.

\section{Acknowledgements}

The author's acknowledge that they have no financial interest in either the HRT II or GDx prototypes.

\section{References}

1 Sommer A, Pollack I, Maumee AE. Optic disc parameters and onset of glaucomatous field loss. Arch Ophthalmol 1979; 97: 1444-1448.

2 Pederson JE, Anderson DR. The mode of progressive disc cupping in ocular hypertension and glaucoma. Arch Ophthalmol 1980; 98: 490-495.

3 Quigley HA, Addicks EM, Green WR. Optic nerve damage in human glaucoma. Arch Ophthalmol 1981; 100: 135-146.

4 Sommer A, Katz J, Quigley HA, Miller NR, Robin AL, Richter RC et al. Clinically detectable nerve fiber atrophy precedes onset of glaucomatous field loss. Arch Ophthalmol 1991; 109: 77-83.

5 Bathija R, Zangwill L, Berry CC, Sample PA, Weinreb RN. Detection of early glaucomatous structural damage with confocal scanning laser tomography. J Glaucoma 1998; 7: 121-127.

6 Mikelberg FS, Parfitt CM, Swindale NV, Graham SL, Drance $\mathrm{SM}$, Gosine R. Ability of the heidelberg retina tomograph to detect early glaucomatous visual field loss. J Glaucoma 1995; 4: $242-247$.

7 Zangwill LM, Bowd C, Berry CC, Williams J, Blumenthal EZ, Sanchez-Galeana CA et al. Discriminating between normal and glaucomatous eyes using the heidelberg retina tomagraph, GDx nerve analyzer, and optical coherence tomograph. Arch Ophthalmol 2001; 119(7): 985-993.

8 Choplin NT, Lundy DC, Dreher AW. Differentiating patients with glaucoma from glaucoma suspects and normal subjects by nerve fiber layer assessment with scanning laser polarimetry. Ophthalmology 1998; 105: 2068-2076.

9 Wollstein G, Garway-Heath DF, Hitchings RA. Identification of early glaucoma cases with the scanning laser ophthalmoscope. Ophthalmology 1998; 105: 1557-1563.

10 Poinoosawmy D, Tan JC, Bunce C, Hitchings R. The ability of the GDx nerve fiber layer analyzer neural network to diagnose glaucoma. Graefe's Arch Clin Exp Ophthalmol 2001; 239: $122-127$

11 Sinai MJ, Essock EA, Fechtner RD, Srinivasan N. Diffuse and localized nerve fiber layer loss measured with a scanning laser polarimeter: sensitivity and specificity of detecting glaucoma. J Glaucoma 2000; 9: 154-162.

12 Kamal DS, Bunce C, Hitchings RA. The use of the GDx to detect retinal nerve fiber layer thickness between normal, ocular hypertension and early glaucoma. Eye 2000; 14: 367-370.

13 Weinreib RN, Zangwill L, Berry CC, Bathija R, Sample PA. Detection of glaucoma with scanning laser polarimetry. Arch Ophthalmol 1998; 116: 1583-1590.

14 Choplin NT, Lundy DC. The sensitivity and specificity of scanning laser polarimetry in the detection of glaucoma in a clinical setting. Ophthalmology 2001; 108: 899-904.

15 Tjon-Fo-Sang M, Lemij HG. The sensitivity and specificity of nerve fiber layer measurements in glaucoma as determined with scanning laser polarimetry. Am J Ophthalmol 1997; 123: 62-69.

16 Iester M, Mikelberg FS, Drance SM. The effect of optic disc size on diagnostic precision with the heidelberg retinal tomograph. Ophthalmology 1997; 104: 545-548.

17 Choplin NT, Schallhorn SC. The effect of excimer laser photorefractive keratotomy for myopia on nerve fiber layer thickness measurement as determined by scanning laser polarimetry. Ophthalmology 1999; 106: 1019-1023.

18 Van Blockland CJ, Verhelst SC. Corneal polarization in the living human eye explained with a biaxial model. J Opt Soc Am 1987; 4: 82-90.

19 Garway-Heath, DF Greaney MJ, Caprioli J. Correction for the erroneous compensation of anterior segment birefringence with the scanning laser polarimeter for glaucoma diagnosis. Invest Ophthalmol Vis Sci 2002; 43(5): 1465-1474.

20 Hoh ST, Greenfields DS, Liebmann JM, Maw R, Ishikawa H, Chew SJ et al. Factors affecting image acquisition during scanning laser polarimetry. Ophthalmic Surg Lasers 1998; 29: 545-551. 